\title{
Importance of fractional exhaled nitric oxide in the differentiation of asthma-COPD overlap syndrome, asthma, and COPD
}

This article was published in the following Dove Press journal:

International Journal of COPD

26 September 2016

Number of times this article has been viewed

\section{Feng-jia Chen* \\ Xin-yan Huang* \\ Yang-li Liu \\ Geng-peng Lin \\ Can-mao Xie}

Department of Respiratory Disease, The First Affiliated Hospital, Sun Yat-sen University, Guangzhou,

People's Republic of China

*These authors contributed equally to this work
Correspondence: Can-mao Xie Respiratory Department, The First Affiliated Hospital of Sun Yat-sen University, Institute of Respiratory Diseases of Sun Yat-Sen University, No 58 Zhongshan 2nd Road, Guangzhou 510080, Guangdong, People's Republic of China Tel +862087755766

Email syshuxineike@।63.com
Background: Fractional exhaled nitric oxide (FeNO) is an easy, sensitive, reproducible, and noninvasive marker of eosinophilic airway inflammation. Accordingly, FeNO is extensively used to diagnose and manage asthma. Patients with COPD who share some of the features of asthma have a condition called asthma-COPD overlap syndrome (ACOS). The feasibility of using FeNO to differentiate ACOS patients from asthma and COPD patients remains unclear. Methods: From February 2013 to May 2016, patients suspected with asthma and COPD through physician's opinion were subjected to FeNO measurement, pulmonary function test (PFT), and bronchial hyperresponsiveness or bronchodilator test. Patients were divided into asthma alone group, COPD alone group, and ACOS group according to a clinical history, PFT values, and bronchial hyperresponsiveness or bronchodilator test. Receiver operating characteristic (ROC) curves were obtained to elucidate the clinical functions of FeNO in diagnosing ACOS. The optimal operating point was also determined.

Results: A total of 689 patients were enrolled in this study: 500 had asthma, 132 had COPD, and 57 had ACOS. The FeNO value in patients with ACOS was 27 (21.5) parts per billion (ppb; median [interquartile range]), which was significantly higher than that in the COPD group (18 [11] ppb). The area under the ROC curve was estimated to be 0.783 for FeNO. Results also revealed an optimal cutoff value of $>22.5 \mathrm{ppb}$ FeNO for differentiating ACOS from COPD patients (sensitivity $70 \%$, specificity $75 \%$ ).

Conclusion: FeNO measurement is an easy, noninvasive, and sensitive method for differentiating ACOS from COPD. This technique is a new perspective for the management of COPD patients.

Keywords: fractional exhaled nitric oxide, chronic obstructive pulmonary disease, asthma, ACOS, cutoff point

\section{Introduction}

Asthma and COPD are common diseases of airways and lungs and share some common clinical characteristics. Asthma, a chronic disorder of the airways, is associated with two main characteristics: airway hyperresponsiveness (AHR) and airway inflammation. The airflow limitation is reversible. ${ }^{1}$ Meanwhile, COPD is a disease characterized by persistent, progressive airflow limitation and is also an inflammatory disease. ${ }^{2}$ Fractional exhaled nitric oxide (FeNO) is an easy, sensitive, reproducible, and noninvasive marker for directly detecting eosinophilic airway inflammation. ${ }^{3}$ Given that airway inflammation can be assessed through FeNO measurement, the American Thoracic Society clinical practice guideline recommends the use of FeNO to diagnose and monitor asthma. ${ }^{4}$ Some patients with COPD have the features of asthma; FeNO values are elevated in this 
specific category of patients with COPD, and this condition is called asthma-COPD overlap syndrome (ACOS). ${ }^{5}$ ACOS was proposed by the science committees of both Global Initiative for Asthma (GINA) and Global Initiative for Chronic Obstructive Lung Disease (GOLD) in 2015. ${ }^{6}$ However, the exact role of FeNO in patients with ACOS remains to be defined. In the present study, we performed a retrospective study that aimed to evaluate the diagnostic performance of FeNO, which may be able to indicate airway eosinophilic inflammation in patients with COPD and to differentiate ACOS from COPD.

\section{Patients and methods}

\section{Patient population}

Patients suspected to have COPD and asthma visiting the First Affiliated Hospital of Sun Yet-Sen University from February 2013 to May 2016 were retrospectively enrolled in this study. Inclusion criteria were physician-diagnosed asthma and COPD. All patients underwent pulmonary function test (PFT) and FeNO test. Subjects were excluded if they had other chronic obstructive lung diseases, including but not limited to bronchiectasis, cystic fibrosis, obliterative bronchiolitis, and reactive airway dysfunction syndrome. Subjects were also excluded if they were current smokers, had a respiratory tract infection within 6 weeks, or used any oral corticosteroid in the previous 4 weeks.

The Institutional Review Board of the First Affiliated Hospital of Sun Yat-sen University approved the study protocol and waived the need for written informed consent from patients due to no intervention trial and the retrospective nature of the study.

\section{Methods}

Patients were divided into three study groups: asthma alone, COPD alone, and ACOS.

COPD patients were diagnosed according to the GOLD criterion, ie, bronchodilator forced expiratory volume in 1 second to forced vital capacity ratio $\left(\mathrm{FEV}_{1} / \mathrm{FVC}\right)$ of $<0.70$. $^{7}$ COPD severity was also classified according to the PFT values and GOLD guidelines. ${ }^{7}$ The included COPD patients were in a stable phase.

Asthma patients were diagnosed according to a clinical history of wheezing, cough, or shortness of breath, as well as the presence of either bronchial hyperresponsiveness or positive broncodilator test $(>12 \%$ and $>200 \mathrm{~mL})$, based on the GINA guidelines. ${ }^{8}$

There are currently no internationally accepted criteria for the diagnosis of ACOS. Thus, in this study, we defined ACOS patients as having a diagnosis of COPD, positive bronchodilator test with $>12 \%$ and $200 \mathrm{~mL}$ gain in $\mathrm{FEV}_{1}$, and presence of clinical characteristics of asthma (previous history of asthma/wheezing).

Medical information, patient characteristics, and data (including height, weight, body surface area, smoking status, pack years of tobacco use, FeNO values, PFT values, and current medication information) were obtained from the patients' medical charts.

\section{Measurements PFT}

PFT was performed using a pneumotachograph-based system in accordance with the recommendations of the European Respiratory Society. ${ }^{9}$ Percentage predicted values (\% pred) were calculated based on previously determined reference values for healthy Chinese adults. All subjects were asked to undergo PFT in a reproducible way, and the best values were retained.

\section{Bronchial hyperresponsiveness test}

PFT values were assessed prior to histamine challenge. Airway responsiveness is expressed as the provocative concentration of histamine required to induce a $20 \%$ decrease in $\mathrm{FEV}_{1}$ (PC20), and for this study, the positive response was defined as PC20 $\leq 8 \mathrm{mg} / \mathrm{mL}$.

\section{Bronchodilator reversibility test}

After baseline evaluation, PFT was repeated 15 minutes after two inhalations of a salbutamol metered dose inhaler through a spacer device. Three forced expiratory maneuvers were obtained, and postbronchodilator $\mathrm{FEV}_{1}$ (\% pred) was recorded. The positive response was defined as improvement in $\mathrm{FEV}_{1}>200 \mathrm{~mL}$ and $>12 \%$ after salbutamol inhalation.

\section{Measurement of FeNO}

FeNO level was measured according to the American Thoracic Society/European Respiratory Society recommendations using an NO analyzer (NIOX MINO Analyzer; Aerocrine AB, Solna, Sweden). Patients were instructed to inhale NO-free air to total lung capacity and immediately exhale fully into the device at a flow rate of $50 \mathrm{~mL} / \mathrm{s}$ for 10 seconds. ${ }^{10}$

\section{Statistical analysis}

All analyses were performed by using SPSS version 18.0 (SPSS Inc., Chicago, IL, USA). Results are expressed as mean \pm standard deviation (SD) for quantitative variables, or median (interquartile range) for non-normal variables when appropriate, or number (\%) of patients. Continuous 
variables were compared using the Student's $t$-test for those that were normally distributed and the Kruskal-Wallis tests for those that were not. The chi-squared test was used to compare categorical variables. Comparison between tests was performed by constructing receiver operating characteristic (ROC) curve and measuring the area under the curve (AUC). The optimal cutoff value was determined, and sensitivity and specificity were also calculated. Statistical significance was defined as $P<0.05$.

\section{Results}

\section{Characteristics of study subjects}

A total of 689 patients were enrolled in this study. According to the diagnostic criterion, they were divided into asthma alone (72.57\%), ACOS (8.27\%), and COPD alone (19.16\%). Most asthma patients had normal PFT values. Patients with asthma showed higher PFT values and FeNO levels than the other two groups $(P<0.001)$. The characteristics of the patients analyzed in this study are listed in Table 1.

\section{Comparison of FeNO levels among the three groups}

There was a significant difference in FeNO levels among the three groups $(P<0.001)$. The FeNO value in patients with

Table I Baseline characteristics of the study population

\begin{tabular}{|c|c|c|c|}
\hline Parameter & $\begin{array}{l}\text { Asthma } \\
(\mathrm{N}=500)\end{array}$ & $\begin{array}{l}\text { COPD } \\
(\mathrm{N}=132)\end{array}$ & $\begin{array}{l}\text { ACOS } \\
(N=57)\end{array}$ \\
\hline lean age, years & $41.92 \pm 15.23$ & $63.52 \pm 9.58$ & $63.17 \pm 9.17$ \\
\hline $\operatorname{ex}(M: F), n$ & & & \\
\hline eight (cm) & $|62.4| \pm 7.35$ & $165.20 \pm 6.98$ & $163.26 \pm 7.70$ \\
\hline Veight (kg) & $59.40 \pm 10.06$ & $60.18 \pm 9.58$ & $59.05 \pm 8.96$ \\
\hline ody surface area $\left(\mathrm{m}^{2}\right)$ & $1.63 \pm 0.15$ & $1.65 \pm 0.15$ & $1.63 \pm 0.14$ \\
\hline VC (\% predicted) & $96.99 \pm 15.59$ & $75.80 \pm 17.73$ & $77.42 \pm 17.56$ \\
\hline $\mathrm{V}_{1}(\%$ predicted $)$ & $88.49 \pm 19.38$ & $50.81 \pm 19.30$ & $51.01 \pm 18.64$ \\
\hline EV//FVC (\%) & $76.89 \pm 11.37$ & $51.44 \pm 12.37$ & $51.88 \pm 11.70$ \\
\hline EF (\% predicted) & 07 & $43.88 \pm 17.06$ & $46.95 \pm 17.27$ \\
\hline EF (\% pred & $75.87 \pm 31$ & & $26.88 \pm 17.72$ \\
\hline IEF25 (\% predicted) & $73.47 \pm 38.75$ & $21.29 \pm|5.4|$ & $26.04 \pm 22.25$ \\
\hline IEF50 (\% predicted) & $70.73 \pm 30.61$ & $24.36 \pm 18.10$ & $24.91 \pm 17.28$ \\
\hline MEF75 (\% predicted) & $75.7 I \pm 27.94$ & $27.89 \pm 17.13$ & $29.35 \pm 21.76$ \\
\hline 1VV (\% predicted) & $86.76 \pm 21.72$ & $53.93 \pm 22.88$ & $51.30 \pm 21.27$ \\
\hline VC (\% predicted) & $91.15 \pm 14.82$ & $71.77 \pm 18.25$ & $73.16 \pm 15.36$ \\
\hline $\begin{array}{l}\text { eNO (ppb), median } \\
\text { nterquartile range) }\end{array}$ & $41.0(45.0)$ & $18(1 \mathrm{I})$ & $27(21.5)$ \\
\hline Smoking history, n (\%) & $129(25.80)$ & $98(74.24)$ & $23(40.35)$ \\
\hline \multicolumn{4}{|c|}{$\begin{array}{l}\text { Notes: Measured pulmonary function values are presented as a percentage of } \\
\text { predictive. Data shown as mean } \pm \text { standard deviation unless indicated otherwise. } \\
\text { Abbreviations: M, male; F, female; FEV, forced expiratory volume in I second; } \\
\text { FVC, forced vital capacity; PEF, peak expiratory flow; MEF, maximal midexpiratory } \\
\text { flow; MEF } 25 \text {, forced expiratory flow after } 25 \% \text { of the FVC; MEF50, forced expiratory } \\
\text { flow after } 50 \% \text { of the FVC; MEF } 75 \text {, forced expiratory flow after } 75 \% \text { of the FV; } \\
\text { MVV, maximal voluntary ventilation; VC, vital capacity; FeNO, fractional exhaled } \\
\text { nitric oxide; ppb, parts per billion; ACOS, asthma-COPD overlap syndrome. }\end{array}$} \\
\hline
\end{tabular}

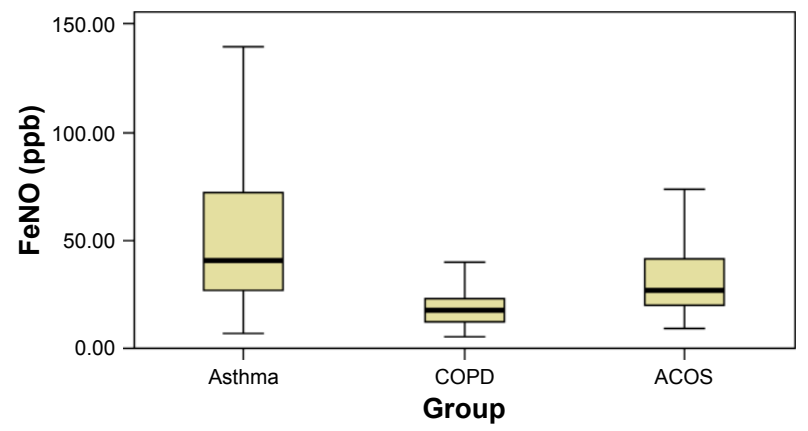

Figure I Comparison of FeNO values among the three groups. Abbreviations: FeNO, fractional exhaled nitric oxide; ppb, parts per billion; ACOS, asthma-COPD overlap syndrome.

ACOS was 27 (21.5) parts per billion (ppb; median [interquartile range]), which was significantly higher than that of the COPD group (18 [11] ppb; $P<0.001)$. The FeNO value in patients with asthma was $41.0(45.0) \mathrm{ppb}$, which was significantly higher than that of the other groups $(P<0.001$; Figure 1)

Figure 2 shows the FeNO values of patients with COPD according to the GOLD severity stage. Differences were observed across the groups in terms of PFT, but no differences existed in FeNO levels among the groups. A total of eight patients $(6.06 \%)$ corresponded to stage I (23.0 [4.5] ppb), 55 (41.67\%) to stage II (14.0 [10.0] ppb), $46(34.85 \%)$ to stage III (18.0 [7.25] ppb), and $23(17.42 \%)$ to stage IV (19.0 [11.0] ppb).

In the ACOS group, $\mathrm{FeNO}$ values were categorized by low $(<25 \mathrm{ppb})$, intermediate $(25-50 \mathrm{ppb})$, and high ( $>50 \mathrm{ppb})$ values, as summarized in Table 2 . The number of cases with low $\mathrm{FeNO}$ values were 25 (43.85\%), with intermediate values were 20 (35.09\%), and with high values were 12 (21.05\%). No differences in PFT values were observed between the groups. The characteristics of patients with ACOS analyzed in this study are listed in Table 2.

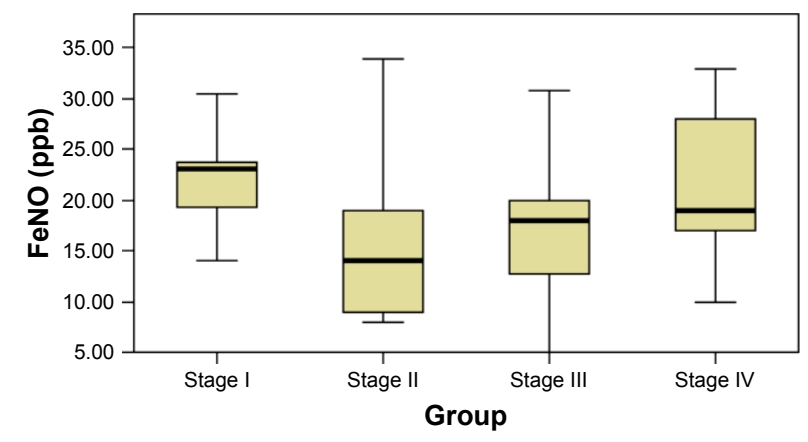

Figure 2 FeNO values by GOLD stage of COPD severity.

Abbreviations: FeNO, fractional exhaled nitric oxide; ppb, parts per billion; GOLD, Global Initiative for Chronic Obstructive Lung Disease. 
Table 2 Comparison of characteristics and PFT values among the groups having low, intermediate, and high FeNO levels

\begin{tabular}{|c|c|c|c|}
\hline Parameter & $\begin{array}{l}\text { FeNO } \\
>\mathbf{5 0} \text { ppb } \\
(\mathbf{N}=12)\end{array}$ & $\begin{array}{l}\mathbf{2 5} \leq \mathrm{FeNO} \\
\leq \mathbf{5 0} \mathbf{p p b} \\
(\mathbf{N}=\mathbf{2 0})\end{array}$ & $\begin{array}{l}\text { FeNO } \\
<\mathbf{2 5} \text { ppb } \\
(\mathbf{N}=\mathbf{2 5})\end{array}$ \\
\hline Mean age, years & $63.25 \pm 9.17$ & $60.40 \pm 9.91$ & $65.28 \pm 8.30$ \\
\hline $\operatorname{Sex}(M: F), n$ & $7: 5$ & $12: 8$ & $18: 7$ \\
\hline Height (cm) & $161.17 \pm 6.04$ & $163.70 \pm 5.82$ & $163.92 \pm 9.58$ \\
\hline Weight (kg) & $57.75 \pm 7.47$ & $58.50 \pm 8.01$ & $60.12 \pm 10.41$ \\
\hline Body surface area $\left(\mathrm{m}^{2}\right)$ & $1.60 \pm 0.11$ & $1.63 \pm 0.11$ & $1.65 \pm 0.17$ \\
\hline FVC (\% predicted) & $72.75 \pm 19.33$ & $77.65 \pm 15.82$ & $79.48 \pm 18.28$ \\
\hline $\mathrm{FEV}_{1}$ (\% predicted) & $51.67 \pm 19.46$ & $50.25 \pm 16.97$ & $51.32 \pm 20.20$ \\
\hline $\mathrm{FEV}_{1} / \mathrm{FVC}(\%)$ & $56.67 \pm 12.77$ & $51.00 \pm 9.56$ & $50.28 \pm 12.55$ \\
\hline PEF (\% predicted) & $45.75 \pm 16.00$ & $48.30 \pm 22.29$ & $46.44 \pm 18.79$ \\
\hline MEF (\% predicted) & $28.42 \pm 18.72$ & $28.95 \pm 19.33$ & $24.48 \pm 16.28$ \\
\hline MEF25 (\% predicted) & $28.00 \pm 22.62$ & $29.08 \pm 27.64$ & $22.08 \pm|6.9|$ \\
\hline MEF50 (\% predicted) & $24.42 \pm 17.88$ & $24.45 \pm 16.93$ & $25.52 \pm 19.11$ \\
\hline MEF75 (\% predicted) & $34.00 \pm 30.84$ & $29.05 \pm 17.44$ & $27.36 \pm 20.32$ \\
\hline MVV (\% predicted) & $57.00 \pm 23.89$ & $52.45 \pm 21.77$ & $47.64 \pm 19.68$ \\
\hline VC (\% predicted) & $72.42 \pm 14.49$ & $70.85 \pm 14.99$ & $75.36 \pm 16.32$ \\
\hline Smoking history, n (\%) & $5(4 I .67)$ & $6(30.00)$ & $12(48.00)$ \\
\hline
\end{tabular}

Notes: Measured pulmonary function values are presented as a percentage of predictive. Data shown as mean \pm standard deviation unless indicated otherwise.

Abbreviations: M, male; $F$, female; $\mathrm{FEV}_{\mathrm{p}}$, forced expiratory volume in I second; FVC, forced vital capacity; PEF, peak expiratory flow; MEF, maximal midexpiratory flow; MEF25, forced expiratory flow after $25 \%$ of the FVC; MEF50, forced expiratory flow after $50 \%$ of the FVC; MEF75, forced expiratory flow after $75 \%$ of the FVC; MVV, maximal voluntary ventilation; VC, vital capacity; FeNO, fractional exhaled nitric oxide; Ppb, parts per billion; ACOS, asthma-COPD overlap syndrome; PFT, pulmonary function test.

\section{Optimal FeNO level to define ACOS}

The constructed ROC curve (Figure 3) defines the optimal cutoff value for FeNO level and was used to differentiate ACOS from COPD. The ROC AUC was estimated to be 0.783 for FeNO. The optimal diagnostic cutoff point was $22.5 \mathrm{ppb}$ (with $70 \%$ sensitivity and $75 \%$ specificity). Table 3 shows the sensitivity and specificity values for different criteria tested.

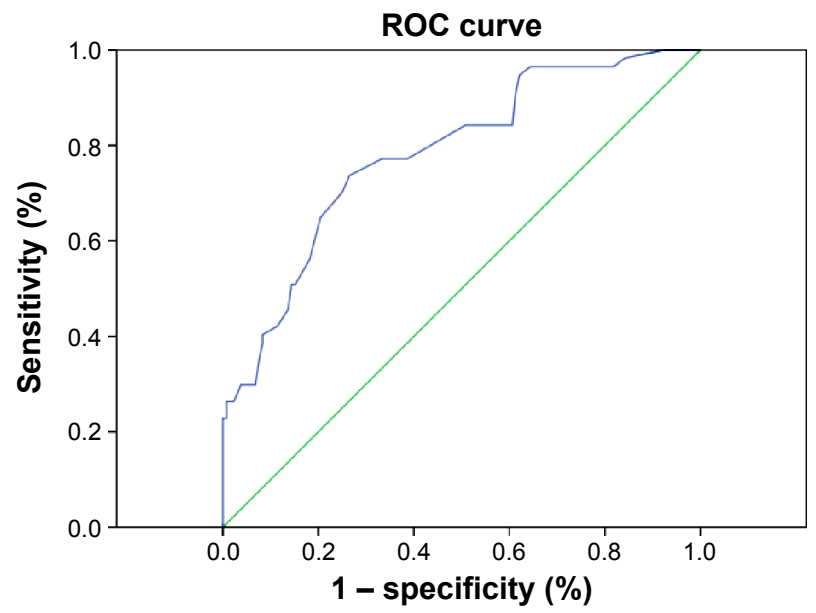

Figure 3 ROC curve analysis of the sensitivity and specificity of FeNO for ACOS diagnosis.

Abbreviations: $\mathrm{ROC}$, receiver operating characteristic; FeNO, fractional exhaled nitric oxide; ACOS, asthma-COPD overlap syndrome.
Table 3 Sensitivity and specificity of different cutoff points used to diagnose ACOS

\begin{tabular}{llll}
\hline & Threshold & Sensitivity & Specificity \\
\hline FeNO value & $>18.5 \mathrm{ppb}$ & 77.2 & 61.4 \\
FeNO value & $>19.5 \mathrm{ppb}$ & 77.2 & 66.7 \\
FeNO value & $>20.5 \mathrm{ppb}$ & 73.7 & 73.5 \\
FeNO value & $>21.5 \mathrm{ppb}$ & 71.9 & 74.2 \\
FeNO value & $>22.5 \mathrm{ppb}$ & 70.2 & 75.0 \\
FeNO value & $>23.5 \mathrm{ppb}$ & 64.9 & 79.5 \\
FeNO value & $>24.5 \mathrm{ppb}$ & 56.1 & 81.8 \\
\hline
\end{tabular}

Abbreviations: ACOS, asthma-COPD overlap syndrome; FeNO, fractional exhaled nitric oxide; ppb, parts per billion.

\section{Discussion}

We performed a retrospective study to define and use FeNO value to differentiate ACOS from asthma and COPD. Results showed that the measurement of FeNO was useful in the diagnosis of ACOS, with an optimal cutoff value of $22.5 \mathrm{ppb}$.

Airway inflammation is considered to be a key element in asthma. In recent years, FeNO has been used as a surrogate marker of eosinophilic inflammation and is a useful tool for the diagnosis and monitoring of asthma. Studies have shown that FeNO is elevated in asthmatic patients and is correlated with airway inflammation. ${ }^{11-13}$ In the present study, FeNO was significantly higher in the asthma group than in the other two groups $(P<0.001)$.

COPD is also an inflammatory disease, but the FeNO value does not differ significantly between patients with COPD and healthy volunteers because neutrophilic inflammation is predominant. ${ }^{14} \mathrm{FeNO}$ values are increased only in a subgroup of patients with COPD, these COPD patients share some common features with asthma. ${ }^{15}$ This condition is called ACOS, whose definition basically depends on the patient's symptoms or the physician's opinion. Many studies have shown that ACOS patients have higher rates of exacerbation, rapid decline in pulmonary function, and poor quality of life compared with those having COPD or asthma alone. ${ }^{16-18}$ These data indicate the importance of finding a biomarker that differentiates ACOS from asthma and COPD as early as possible. A number of preliminary studies have measured FeNO in COPD, but literature defining the role of FeNO in patients with ACOS is minimal. In the present study, we found that the FeNO values were substantially higher in COPD patients with ACOS than in those with COPD alone $(P<0.01)$. These results confirmed a new perspective concerning the potential role of $\mathrm{FeNO}$ as a biomarker.

Our results showed that 57 patients had a diagnosis of both asthma and COPD, and the prevalence of ACOS was $\sim 30.16 \%$ in patients with COPD. This finding was consistent with the number reported by other investigators 
who suggested that ACOS exists in $15 \%-45 \%$ of COPD patients. ${ }^{17,19,20} \mathrm{We}$ compared the features of patients with ACOS, COPD, and asthma. Patients with asthma were younger than those in the other groups. The PFT values for patients with asthma were better than those in the other groups. PFT values did not significantly differ between the COPD and the ACOS groups $(P>0.05)$. It was easy to differentiate ACOS from asthma because of the clinical characteristics and PFT values, but it was difficult to differentiate ACOS from COPD through PFT values.

Moreover, the mean age of patients with COPD was $63.52 \pm 9.58$ years (mean \pm standard deviation), and the percentages of patients with GOLD stages I, II, III, and IV were $6.06 \%, 41.67 \%, 34.85 \%$, and $17.42 \%$, respectively. We also observed that FeNO values were not linearly associated with GOLD classification, similar to that described by other researchers. ${ }^{14,21}$

FeNO is a good predictor for differentiating ACOS from COPD, with an ROC AUC of 0.783 . Taking $22.5 \mathrm{ppb}$ as a cutoff point to diagnose ACOS, FeNO measurement indicated a sensitivity of $70 \%$ and a specificity of $75 \%$, which agreed with previous studies. Donohue et a $1{ }^{14}$ showed an FeNO level of $20 \mathrm{ppb}$ in COPD patients with a previous diagnosis of asthma. Some authors have observed that patients with a cutoff point of $35 \mathrm{ppb}$ can be classified as ACOS, whether or not they have allergic rhinitis. ${ }^{15}$ Corticosteroids are known to increase the risk for pneumonia and other diseases in COPD patients, ${ }^{14,22}$ but ACOS patients positively respond to treatment with inhaled corticosteroids. Given that FeNO could differentiate ACOS from COPD, FeNO could offer additional advantages for the management of COPD patients.

This is a retrospective study and several limitations exist. First, the diagnosis was confirmed by PFT, which may have yielded false-positive and -negative results. Second, FeNO is a biomarker whose values are influenced by many factors, such as atopy. We made the best attempt to exclude subjects with other diseases that may influence the FeNO levels. We further excluded current smokers, but not ex-smokers, whose NO production may have been affected. Although the conclusions about the relevance of FeNO levels and smoking history in different COPD studies are contradictory. Wagener et $\mathrm{al}^{23}$ reported that smoking history does not affect FeNO, while Kharitonov et $\mathrm{al}^{24}$ reported that current smokers have lower levels of exhaled NO. We do not think the conclusion would be biased by the exclusion of current smokers, but we should admit that the optimal cutoff point and the sensitivity and specificity of FeNO may be different if we had enrolled current smokers. Finally, although we have tried vigorously to standardize the method of FeNO testing, we should be cautious in extrapolating absolute values obtained in this center to those obtained in other centers.

\section{Conclusion}

We found that FeNO measurement is an alternative diagnostic tool to conventional lung function tests for diagnosing asthma. The majority of patients with COPD do not exhibit high FeNO levels, but a high FeNO level of a COPD patient may predict that this patient has ACOS. FeNO measurement is a simple, reproducible, and noninvasive method of differentiating ACOS from COPD, which may have implications in the management of patients with COPD. However, we need additional large-scale prospective studies to expand the clinical application of FeNO measurement.

\section{Disclosure}

The authors report no conflicts of interest in this work.

\section{References}

1. Chung KF, Wenzel SE, Brozek JL, et al. International ERS/ATS guidelines on definition, evaluation and treatment of severe asthma. Eur Respir J. 2014;43(2):343-373.

2. Vestbo J, Anderson W, Coxson HO, et al. Evaluation of COPD longitudinally to identify predictive surrogate end-points (ECLIPSE). Eur Respir J. 2008;31:869-873.

3. Alving K, Malinovschi A. Basic aspects of exhaled nitric oxide. In: Horvath I, de Jongste JC, editors. Exhaled Biomarkers. European Respiratory Society Monographs. Sheffield: European Respiratory Society; 2010:1-31.

4. Dweik RA, Boggs PB, Erzurum SC, et al. An official ATS clinical practice guideline: interpretation of exhaled nitric oxide levels (FENO) for clinical applications. Am J Respir Crit Care Med. 2011;184(5): 602-615.

5. Drazen JM, Postma DS, Rabe KF. The asthma-COPD overlap syndrome. N Engl J Med. 2015;373(13):1241-1249.

6. Global Initiative for Asthma [webpage on the Internet]. Diagnosis of Diseases of Chronic Airflow Limitation: Asthma COPD and AsthmaCOPD Overlap Syndrome (ACOS). Available from: http://www. ginasthma.org/local/uploads/files/ACOS_2015.pdf [updated 2015]. Accessed May 7, 2015.

7. The Global Strategy for the Diagnosis, Management and Prevention of COPD [webpage on the Internet]. Global Initiative for Chronic Obstructive Lung Disease (GOLD) 2013. Available from: http://www. goldcopd.org/. Accessed April 22, 2014.

8. Global Initiative for Asthma. Global strategy for asthma management and prevention [revised 2010]. Available from: www.ginasthma.org. Accessed March 16, 2012.

9. Miller MR, Hankinson J, Brusasco V, et al. Standardisation of spirometry. Eur Respir J. 2005;26:319-338.

10. American Thoracic Society, European Respiratory Society. ATS/ERS recommendations for standardized procedures for the online and offline measurement of exhaled lower respiratory nitric oxide and nasal nitric oxide, 2005. Am J Respir Crit Care Med. 2005;171(8):912-930.

11. Al-Ali MK, Eames C, Howarth PH. Exhaled nitric oxide: relationship to clinicophysiological markers of asthma severity. Respir Med. 1998;92:908-913.

12. Jatakanon A, Lim S, Kharitonov SA, Chung KF, Barnes PJ. Correlation between exhaled nitric oxide,sputim eosinophils, and methacholine responsiveness in patients with mild asthma. Thorax. 1998;53:91-95. 
13. Lim S, Jatakanon A, Meah S, Oates T, Chung KF, Barnes P. Relationship between exhaled nitric oxide and mucosal eosinophilc inflammation in mild to moderately severe asthma. Thorax. 2000;55:184-188.

14. Donohue JF, Herje N, Crater G, Rickard K. Characterization of airway inflammation in patients with COPD using fractional exhaled nitric oxide levels: a pilot study. Int J Chronic Obstructive Pulm Dis. 2014;9: 745-751.

15. Tamada T, Sugiura H, Takahashi T, et al. Biomarker-based detection of asthma-COPD overlap syndrome in COPD populations. Int J Chronic Obstructive Pulm Dis. 2015;10:2169-2176.

16. Kauppi $P$, Kupiainen H, Lindqvist A, et al. Overlap syndrome of asthma and COPD predicts low quality of life. J Asthma. 2011;48:279-285.

17. Gibson PG, Simpson JL. The overlap syndrome of asthma and COPD: what are its features and how important is it? Thorax. 2009;64: 728-735.

18. Andersén H, Lampela $P$, Nevanlinna $A$, Sänääangas $O$, Keistinen T. High hospital burden in overlap syndrome of asthma and COPD. Clin Respir J. 2013;7:342-346.

19. Soriano JB, Davis KJ, Coleman B, Visick G, Mannino D, Pride NB. The proportional Venn diagram of obstructive lung disease: two approximations from the United States and the United Kingdom. Chest. $2003 ; 124(2): 474-481$
20. Beeh KM, Kornmann O, Beier J, Ksoll M, Buhl R. Clinical application of a simple questionnaire for the differentiation of asthma and chronic obstructive pulmonary disease. Respir Med. 2004;98(7):591-597.

21. Alcázar-Navarrete B, Romero-Palacios PJ, Ruiz-Sancho A, RuizRodriguez O. Diagnostic performance of the measurement of nitric oxide in exhaled air in the diagnosis of COPD phenotypes. Nitric Oxide. 2016;54:67-72.

22. Karampitsakos T, Gourgoulianis KI.Asthma-COPD Overlap Syndrome (ACOS): single disease entity or not? Could exhaled nitric oxide be a useful biomarker for the differentiation of ACOS, asthma and COPD? Med Hypotheses. 2016;91:20-23.

23. Wagener AH, De Nijs SB, Lutter R, et al. External validation of blood eosinophils, FENO and serum periostin as surrogates for sputum eosinophils in asthma. Thorax. 2015;70:115-120.

24. Kharitonov SA, Robbins RA, Yates D, Keatings V, Barnes PJ. Acute and chronic effects of cigarette smoking on exhaled nitric oxide. Am J Respir Crit Care Med. 1995;152(2):609-612.

\section{Publish your work in this journal}

The International Journal of COPD is an international, peer-reviewed journal of therapeutics and pharmacology focusing on concise rapid reporting of clinical studies and reviews in COPD. Special focus is given to the pathophysiological processes underlying the disease, intervention programs, patient focused education, and self management protocols.

\section{Dovepress}

This journal is indexed on PubMed Central, MedLine and CAS. The manuscript management system is completely online and includes a very quick and fair peer-review system, which is all easy to use. Visit http://www.dovepress.com/testimonials.php to read real quotes from published authors. 